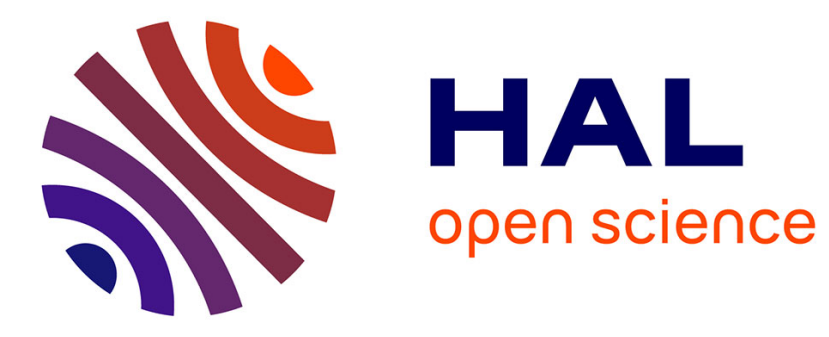

\title{
Les Acrididae des îles Eparses (Orthoptera, Caelifera)
}

Raphaël Parnaudeau, Jacques Rochat, Antoine Franck, Sophie Gasnier, Grégory Cazanove, Sylvain Hugel

\section{To cite this version:}

Raphaël Parnaudeau, Jacques Rochat, Antoine Franck, Sophie Gasnier, Grégory Cazanove, et al.. Les Acrididae des îles Eparses (Orthoptera, Caelifera). Bulletin de la Société Entomologique de France, 2013, 118 (1), pp.111-117. 10.3406/bsef.2013.2590 . hal-03431837

\section{HAL Id: hal-03431837 https://hal.science/hal-03431837}

Submitted on 16 Nov 2021

HAL is a multi-disciplinary open access archive for the deposit and dissemination of scientific research documents, whether they are published or not. The documents may come from teaching and research institutions in France or abroad, or from public or private research centers.
L'archive ouverte pluridisciplinaire $\mathbf{H A L}$, est destinée au dépôt et à la diffusion de documents scientifiques de niveau recherche, publiés ou non, émanant des établissements d'enseignement et de recherche français ou étrangers, des laboratoires publics ou privés. 


\section{Les Acrididae des îles Eparses (Orthoptera, Caelifera)}

Raphaël Parnaudeau, Jacques Rochat, Antoine Franck, Sophie Gasnier, Grégory Cazanove, Sylvain Hugel

\section{Résumé}

Les îles Eparses constituent une unité administrative regroupant des îlots français de basse altitude du sud-ouest de l'océan Indien. A ce jour, sept espèces d'Acrididae étaient signalées sur au moins une de ces îles. Une série de missions menées par le Muséum d'Histoire naturelle de la Réunion, l'Insectarium de la Réunion et le CIRAD-Réunion, permettent de compléter l'inventaire des Acrididae de ces îles. Quatre espèces additionnelles sont signalées sur au moins une de ces îles : Diabolocatantops axillaris saucius (Burmeister, 1838), Acrida madecassa (Brancsik, 1893), Acrotylus patruelis (Herrich-Schäffer, 1838) et Oedaleus virgula (Snellen van Vollenhoven, 1870).

\section{Abstract}

Acrididae from the Scattered Islands (Orthoptera, Caelifera). The Scattered Islands are an administrative entity grouping French low altitude islets in South Western Indian Ocean. Until now, seven Acrididae were known from at least one of these islands. Fieldworks organized by the Muséum d'Histoire naturelle de la Réunion, the Insectarium de la Réunion and the CIRAD-Réunion, allowed updating this checklist. Four additional species were recorded from at least one of these islands : Diabolocatantops axillaris saucius (Burmeister, 1838), Acrida madecassa (Brancsik, 1893), Acrotylus patruelis (Herrich-Schäffer, 1838) and Oedaleus virgula (Snellen van Vollenhoven, 1870).

\section{Citer ce document / Cite this document :}

Parnaudeau Raphaël, Rochat Jacques, Franck Antoine, Gasnier Sophie, Cazanove Grégory, Hugel Sylvain. Les Acrididae des îles Eparses (Orthoptera, Caelifera). In: Bulletin de la Société entomologique de France, volume 118 (1),2013. pp. 111-117;

https://www.persee.fr/doc/bsef_0037-928x_2013_num_118_1_2590

\section{Ressources associées :}

Acrididae 


\title{
Les Acrididae des îles Eparses (Orthoptera, Caelifera)
}

\author{
par Raphaël Parnaudeau ${ }^{1}$, Jacques Rochat ${ }^{2}$, Antoine FrancK ${ }^{3}$, \\ Sophie GASNIER ${ }^{2}$, Grégory CAZANOve ${ }^{4} \&$ Sylvain HugEL ${ }^{5 *}$ \\ ${ }^{1}$ Société Réunionnaise des Amis du Muséum, 1 rue Poivre, F - 97400 Saint-Denis de la Réunion \\ $<$ r.parn@orange.fr> \\ ${ }^{2}$ Insectarium de la Réunion, rue Simon-Pernic, Pépinière Communale, F - 97420 Le Port \\ $<$ contact@insectarium-reunion.com> \\ ${ }^{3}$ CIRAD, 7 chemin de l'IRAT, Ligne Paradis, F - 97410 Saint-Pierre <antoine.franck@cirad.fr> \\ ${ }^{4}$ Muséum d'Histoire naturelle de la Réunion, Jardin de l'Etat, 1 rue Poivre, F - 97400 Saint-Denis \\ <gregory.cazanove@cg974.fr> \\ ${ }^{5}$ UPR 3212 CNRS, Université de Strasbourg, 21 rue René-Descartes, F - 67084 Strasbourg cedex \\ $<$ hugels@inci-cnrs.unistra.fr $>*$ correspondance
}

Résumé. - Les îles Eparses constituent une unité administrative regroupant des îlots français de basse altitude du sud-ouest de l'océan Indien. A ce jour, sept espèces d'Acrididae étaient signalées sur au moins une de ces îles. Une série de missions menées par le Muséum d'Histoire naturelle de la Réunion, l'Insectarium de la Réunion et le CIRAD-Réunion, permettent de compléter l'inventaire des Acrididae de ces îles. Quatre espèces additionnelles sont signalées sur au moins une de ces îles : Diabolocatantops axillaris saucius (Burmeister, 1838), Acrida madecassa (Brancsik, 1893), Acrotylus patruelis (Herrich-Schäffer, 1838) et Oedaleus virgula (Snellen van Vollenhoven, 1870).

Abstract. - Acrididae from the Scattered Islands (Orthoptera, Caelifera). The Scattered Islands are an administrative entity grouping French low altitude islets in South Western Indian Ocean. Until now, seven Acrididae were known from at least one of these islands. Fieldworks organized by the Muséum d'Histoire naturelle de la Réunion, the Insectarium de la Réunion and the CIRAD-Réunion, allowed updating this checklist. Four additional species were recorded from at least one of these islands: Diabolocatantops axillaris saucius (Burmeister, 1838), Acrida madecassa (Brancsik, 1893), Acrotylus patruelis (Herrich-Schäffer, 1838 ) and Oedaleus virgula (Snellen van Vollenhoven, 1870).

Keywords. - Islands, Mascarene, Madagascar, Comoros, Seychelles, grasshoppers, locusts.

Les îles Eparses constituent une unité administrative regroupant des îles de basse altitude du sud-ouest de l'océan Indien : Bassas-da-India, Europa, Glorieuses (Grande Glorieuse et île du Lys), Juan de Nova et Tromelin. Bassas-da-India étant presque totalement submersible, elle n'est pas considérée dans le présent article. Ces îles ont en commun d'avoir été submergées lors des périodes interglaciaires, leur faune et flore terrestres résultent donc d'une colonisation relativement récente. De nombreux Acrididae des territoires voisins vivent dans des formations herbeuses ou buissonnantes et présentent une capacité de dispersion importante. De tels Acrididae semblent idéalement adaptés pour atteindre et s'établir sur ces îles.

A ce jour, sept espèces d'Acrididae étaient signalées sur au moins une de ces îles (PAULIAN, 1950, 1951 ; VIETTE, 1967 ; DirSh \& DescAmPs, 1968 ; $c f$. tableau I) : Catantopsis malagassus Karny, 1902, Catantopsis sacalava Brancsik, 1893, Cyrtacanthacris aeruginosa (Stoll, 1813) probablement confondu avec l'espèce suivante, Cyrtacanthacris tatarica (Linné, 1758), Rhadinacris schistocercoides (Brancsik, 1893), Aiolopus thalassinus rodericensis (Butler, 1876) et Conipoda calcarata (Saussure, 1884).

Une série de missions ont été conduites par le Muséum d'Histoire naturelle de la Réunion aux Glorieuses (2003, 2004), à Europa (2002), à Juan de Nova (2003, 2006, 2007) et à Tromelin (2003), complétées par des collectes opportunistes durant les missions du laboratoire ECOMAR 
(Université de la Réunion) à Juan de Nova (2007) et Europa (2008). Dans le cadre du programme de recherches du Conservatoire national botanique de Mascarins (La Réunion), les auteurs ont pu joindre la mission scientifique pluridisciplinaire du navire Marion Dufresne dans les îles Eparses et réaliser en avril 2011 des relevés faunistiques approfondis sur l'ensemble des îles. Ces missions permettent d'actualiser les connaissances sur les Acrididae des îles Eparses. Dans le présent article, quatre espèces additionnelles sont signalées sur au moins une des îles : Diabolocatantops axillaris saucius (Burmeister, 1838), Acrida madecassa (Brancsik, 1893), Acrotylus patruelis (Herrich-Schäffer, 1838) et Oedaleus virgula (Snellen van Vollenhoven, 1870). Aucun Acrididae n'a été observé à Tromelin ni lors de la mission menée par le muséum (15 décembre 2003) ni précédemment (Paulian, 1955). La distribution des Criquets dans les autres îles de la région est discutée.

Méthodes. - Les collectes ont été réalisées pour la plupart à vue de jour et de nuit ou à l'aide d'un filet fauchoir. Deux espèces ont été attirées par les lampes de pièges lumineux : Aiolopus thalassinus rodericensis et Catantopsis malagassus. Cette méthode pourrait d'ailleurs être mise à profit afin d'étudier ces espèces et peut-être de retrouver Catantopsis sacalava aux Glorieuses.

Les identifications ont été effectuées par S. Hugel sur des spécimens adultes.

Abréviations des institutions. - MHNG : Muséum d'Histoire Naturelle de Genève ; MNHN : Muséum national d'Histoire naturelle, Paris ; MHN RUN : Muséum d'Histoire Naturelle de la Réunion.

Abréviations des collecteurs. - AF : Antoine Franck ; GC : Grégory Cazanove ; JD : Jean David ; JR : Jacques Rochat ; RP : Raphaël Parnaudeau ; R\&D : Sonia Ribes et Patrick Durville ; SG : Sophie Gasnier.

\section{RÉSULTATS (voir aussi tableaux I et II)}

Diabolocatantops axillaris saucius (Burmeister, 1838)

ILE DU Lys, GLORIEUSES : 1 ô, 19.V.2003, RP leg., MHN RUN (Ins. 4136) ; 1 क , 19.V.2003, plage, $R P$ leg., MHN RUN (Ins. 4138) ; 1 +, 19.V.2004, RP leg., MHN RUN (Ins. 5061) ; 1 + , 19.V.2003, RP leg., MHN RUN (Ins. 4442) ; 1 o, 19.V.2003, RP leg., MHN RUN (Ins. 4441) ; 1 ㅇ, 19.V.2003, $R P$ leg., MHN RUN (Ins. 4438) ; $\hat{O}$ O , 20.IV.2011, à vue, SG leg., MHN RUN (2011.E.4.1.52) ; 1 क, idem, MHN RUN (2011.E.4.1.53) ; 19, idem, sur Tournefortia argentea L., à vue, GC leg., MHN RUN (2011.E.4.1.50) ; 1 ㅇ, idem, sur Cordia sp., à vue, SG leg., MHN RUN (2011.E.4.3.19).

GRANDE GloRIEUSE : 1 क, 23.V.2003, RP leg., prairie, piste d'aviation, MHN RUN (Ins. 4439) ; 1 ô, 23.V.2003, RP leg., MHN RUN (Ins. 4449) ; 1 §̂, 23.V.2003, littoral sud, RP leg., MHN RUN (Ins. 4440) ; 1 q, piste, 17.IV.2011, à vue, SG leg., MHN RUN (2011.E.3.3.97) ; 2 §, idem, MHN RUN (2011.E.3.8.32) ; 1 ઈ, idem, 18.IV.2011, sur Guettarda speciosa L., à vue, GC leg., MHN RUN (2011.E.3.4.101).

Catantopsis malagassus Karny, 1907

GRANDE GLORIEUSE : 1 ઈ, 12.II.2003, lumière, RP leg., MHN RUN (Ins. 4437).

Catantopsis sacalava Brancsik, 1893

JUAN DE Nova : 1 ex., 26.VII.2006, E57, 13h45, sur le sol sableux, Peck leg., MHN RUN (Ins. 8008); 1 Oे, 10.IV.2011, piège lumineux, AF leg., MHN RUN (2011.E.2.1.70) ; 1 +, 11.IV.2011, sur Caesalpinia bonduc (L.) Roxb., battage, JR leg., MHN RUN (2011.E.2.4.77) ; 1 + , idem, à vue, AF leg., MHN RUN (2011.E.2.4.98).

Cette espèce est signalée aux Glorieuses (Paulian, 1989). Nous ne l'avons pas observée lors des missions effectuées dans cet archipel.

Cyrtacanthacris aeruginosa (Stoll, 1813)

Cette espèce est citée de la Grande Glorieuse (Paulian, 1989). Nous ne l'avons pas retrouvée lors des différentes missions et aucun spécimen de cette espèce provenant des îles 
Eparses n'est présent au MNHN. C. aeruginosa est une espèce d'Afrique continentale. Comme Cyrtacanthacris tatarica est présent en Grande Glorieuse, il nous semble probable que les Cyrtacanthacris signalés par PAULIAN (1989) correspondent à C. tatarica (voir ci-dessous).

\section{Cyrtacanthacris tatarica (Linné, 1758)}

JuAn DE Nova : 1 ex., 20-21.III.2003, RP leg., MHN RUN (Ins. 4141) ; 1 ex., 23.VII.2006, E34, près de la piste d'atterrissage, Peck leg., MHN RUN (Ins. 8002) ; 1 ex., 26.VII.2006, Peck leg., E61, MHN RUN (Ins. 8003) ; 1 ㅇ, 11.VI.2007, pointe ouest, $R \& D$ leg., MHN RUN (Ins. 6937) ; 1 + 11.VI.2007, maison Patureau, $R \& D$ leg., MHN RUN (Ins. 6938) ; 1 + , IV.2011, à vue, JD leg., MHN RUN (2011.E.2.9.42) ; 1 \%, 13.IV.2011, à vue, $A F$ leg., MHN RUN (2011.E.2.4.38).

Grande Glorieuse : 1 ô, 23.V.2003, prairie piste d'aviation, RP leg., MHN RUN ; 17.V.2004, RP leg., MHN RUN (Ins. 5964).

EUROPA : 1 ex., 11.XII.2002, savane, sentier nord (PK2), RP leg., MHN RUN (Ins. 3300) ; 1 ex., V.1993, Le Corre leg., MHN RUN (Ins. 6844) ; 1 ㅇ, 5.IV.2011, herbe, filet, $A F$ leg., MHN RUN (2011.E.1.2.3) ; 1 +, IV.2011, JD leg., MHN RUN (2011.E.1.8.1) ; 1 §ै, piste station militaire vers rondpoint des Sapeurs, 6.IV.2011, JD leg., MHN RUN (2011.E.1.8.4).

\section{Rhadinacris schistocercoides (Brancsik, 1893)}

EUROPA : 1 ex., 11.XII.2002, savane PK12, 22²0,489’S - 40²2,108’E, RP leg., MHN RUN ; 1 วิ, 5.IV.2011, herbe, filet, $A F$ leg., MHN RUN (2011.E.1.2.2) ; 1 Ô, 6.IV.2011, à vue, $A F$ leg., MHN RUN (2011.E.1.3.4).

\section{Acrida madecassa (Brancsik, 1893)}

JUAN DE Nova : 1 ex., 20-21.III.2003, RP leg., MHN RUN (Ins. 4135) ; 1 +, idem, MHN RUN (Ins. 5870) ; 1 ex., 26.VII.2006, E58, grandes herbes près de la gendarmerie, Peck leg., MHN RUN (Ins. 8001) ; 2 क, 11.VI.2007, dans les herbes autour du lac, $R \& D$ leg., MHN RUN (Ins. 6919, Ins. 6920) ; 2 J, 11.IV.2011, sur Sclerodactylon macrostachyum (Benth.) A. Camus, Daknopholis boivinii (A. Camus) Clayton, Dactyloctenium ctenioides (Steud.) Lorch ex Bosser, fauchage, AF leg., MHN RUN (2011.E.2.2.17) ; 1 ㅇ, piste du Trou Bleu, 12.IV.2011, à vue, $A F$ leg., MHN RUN (2011.E.2.9.22).

Très commun dans les hautes herbes du littoral.

\section{Acrotylus patruelis (Herrich-Schäffer, 1838)}

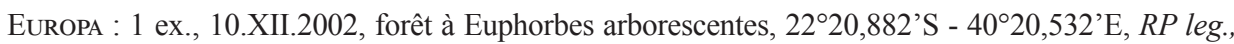
MHN RUN (Ins. 3405) ; 1 ${ }^{1}$, piste station militaire vers rond-point des Sapeurs, 6.IV.2011, JD leg., MHN RUN (2011.E.1.8.2).

Cette espèce géophile se rencontre sur les sentiers de la forêt d'Euphorbes.

Aiolopus thalassinus rodericensis (Butler, 1876)

EUROPA : 2 ex.,11.XII.2002, sentier nord (PK2), 22²0,488'S - 40²2,107’E, RP leg., MHN RUN (Ins. 3367), MHN RUN (Ins. 3368) ; 1 + , 4.IV.2011, piège lumineux, $A F / J R$ leg., MHN RUN (2011.E.1.1.4) ; 1 ô, idem, MHN RUN (2011.E.1.1.5) ; 1 ô, 5.IV.2011, herbe, filet, $A F$ leg., MHN RUN (2011.E.1.2.1) ; 2

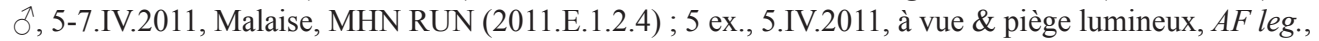
MHN RUN (2011.E.1.3.6) ; 4 ex., piste station militaire vers rond-point des Sapeurs, 6.IV.2011, JD leg., MHN RUN (2011.E.1.8.5).

Grande Glorieuse : 2 ex., 20.II.2003, littoral sud, RP leg., MHN RUN (Ins. 4133), MHN RUN (Ins. 4140) ; 3 ex., 20.V.2003, lumière, RP leg., MHN RUN (Ins. 4132), MHN RUN (Ins. 4139), MHN RUN (Ins. 4137) ; 1 ex., 25.V.2003, RP leg., MHN RUN (Ins. 4161) ; $1 \hat{\jmath}$, piste 17.IV.2011, piège lumineux, SG leg., MHN RUN (2011.E.3.1.201).

JuAn DE Nova : 1 ex., 20-21.III.2003, RP leg., MHN RUN (Ins. 4134) ; 1 ex., 23.VII.2006, E35, près de la piste d'atterrissage, Peck leg. MHN RUN (Ins. 8007) ; 1 + , 11.IV.2011, sur Dactyloctenium sp., 
fauchage, $A F$ leg., MHN RUN (2011.E.2.3.62) ; 1 , idem, fauchage, $A F$ leg., MHN RUN (2011.E.2.2.34) ; 1 §, 13.IV.2011, malaise, JR leg., MHN RUN (2011.E.2.1.152).

Ce taxon est signalé comme Aiolopus sp. (Paulian, 1950), Aiolopus sansibarus (Paulian, 1951) et Aiolopus sansibara (PAUlian, 1989). Les spécimens correspondant sont au MNHN et appartiennent à Aiolopus thalassinus rodericensis $(\mathrm{SH})$.

Oedaleus virgula (Snellen van Vollenhoven, 1870)

Grande Glorieuse : 1 ex., 23.V.2003, dune arrière littoral, nord de l'île, RP leg., MHN RUN (Ins. 4131); 1 , 19.V.2003, RP leg., MHN RUN (Ins. 4436).

JUAN DE NOva : 1 q, 10.VI.2007, Pointe ouest, $R \& D$ leg., MHN RUN (Ins. 6953) ; 1 q, 10.VI.2007, pointe ouest, $R \& D$ leg., MHN RUN (Ins. 6954).

\section{Conipoda calcarata (Saussure, 1884)}

GRANDE GLORIEUSE : 1 ex., 23.V.2003, dune arrière littoral, nord de l'île, RP leg., MHN RUN (Ins. 4113) ; 1 đ , 23.V.2003, dune arrière littoral, nord de l'île, RP leg., MHN RUN (Ins. 4435).

C. calcarata partage le même habitat qu'Oedaleus virgula : les dunes de l'arrière littoral au nord de la Grande Glorieuse.

Une espèce voisine a été décrite d'Aldabra : Conipoda aldabrae Saussure, 1899. Cette espèce a été ensuite signalée sur d'autres îles basses des Seychelles : Cosmoledo et Assomption (Bolívar, 1912 ; Матуot, 1998). Ni les descriptions de Saussure (1884, 1899), ni des photographies détaillées des spécimens-types des deux espèces (MHNG) ne permettent de distinguer ces espèces qui sont peut-être synonymes. Seul un examen direct des spécimenstypes et de séries de spécimens des localités-types permettra de déterminer si Conipoda aldabrae est un synonyme de Conipoda calcarata. Nous décidons pour l'instant d'identifier les spécimens des îles Eparses sous le nom de Conipoda calcarata, suivant en cela l'identification de Dirsh \& Descamps (1968) de spécimen(s) de Juan de Nova.

\section{DisCUSSION}

Diversité des espèces. - Cette étude permet de signaler quatre espèces inédites et de compléter les connaissances sur la répartition des espèces dans chacune des îles (tableau I).

A ce jour, sept Acrididae étaient signalés des îles éparses. Cyrtacanthacris aeruginosa a probablement été confondu avec $C$. tatarica, réduisant à six le nombre d'espèces connues de ces îles. Hormis cette espèce douteuse, nous avons retrouvé toutes les espèces signalées sur chacune des îles excepté $C$. sacalava aux Glorieuses et $C$. calcarata à Juan de Nova.

Malgré sa superficie relativement importante et ses habitats diversifiés, Europa semble moins riche en espèces que les autres îles. Ainsi, l'archipel des Glorieuses et Juan de Nova voient leurs inventaires enrichis de quatre espèces chacun contre une seule nouvelle espèce pour Europa. Il est néanmoins possible que cette île n'ait pas été assez échantillonnée et que l'inventaire des Criquets y soit à compléter.

La spécificité des îles est peu marquée (tableau I). En effet, une espèce ne se rencontre que sur Juan de Nova (Acrida madecassa), une seule n'est présente que sur les Glorieuses (Diabolocatantops axillaris saucius) et deux ne sont présentes que sur Europa (Rhadinacris schistocercoides, Acrotylus patruelis).

La totalité des espèces signalées historiquement des îles Eparses a été retrouvée à l'exception de Catantopsis sacalava aux Glorieuses et Conipoda calcarata à Juan de Nova. Cela semble indiquer une certaine stabilité des populations sur quelques décennies. L'absence d'inventaire régulier empêche de mettre en évidence d'éventuelles colonisations récentes. 
Tableau I. - Acrididae des îles Eparses. Les nouvelles données pour chaque île sont indiquées par "X" ; les espèces retrouvées par "*". Aucune espèce n'est connue à ce jour de Tromelin.

\begin{tabular}{|c|c|c|c|c|}
\hline & Europa & Grande Glorieuse & $\begin{array}{l}\text { Ile du Lys } \\
\text { (Glorieuses) }\end{array}$ & Juan de Nova \\
\hline Catantopsis sacalava & & Paulian, $1989: 205$ & & $\begin{array}{c}\text { DIRSH \& DESCAMPS, } \\
1968: 186 \\
*\end{array}$ \\
\hline Catantopsis malagassus & & $\begin{array}{c}\text { DIRSH \& DESCAMPS, } \\
1968: 187-188 \\
* \\
\end{array}$ & & \\
\hline $\begin{array}{l}\text { Diabolocatantops } \\
\text { axillaris saucius }\end{array}$ & & $\mathrm{X}$ & $\mathrm{X}$ & \\
\hline $\begin{array}{l}\text { Cyrtacanthacris } \\
\text { aeruginosa }\end{array}$ & & $\begin{array}{c}\text { PAULIAN, } 1989: 205 . \\
\text { Probablement } C . \\
\text { tatarica (voir texte). } \\
*\end{array}$ & & \\
\hline Cyrtacanthacris tatarica & $\begin{array}{l}\text { Paulian, } 1950: 84 \\
\text { Viette, } 1967: 195 \\
*\end{array}$ & $\mathrm{X}$ & & $\mathrm{X}$ \\
\hline $\begin{array}{l}\text { Rhadinacris } \\
\text { schistocercoides }\end{array}$ & $\begin{array}{l}\text { Paulian, } 1951: 186 \\
\text { Viette, } 1967: 195 \\
*\end{array}$ & & & \\
\hline Acrida madecassa & & & & $\mathrm{X}$ \\
\hline Acrotylus patruelis & $\mathrm{X}$ & & & \\
\hline $\begin{array}{l}\text { Aiolopus thalassinus } \\
\text { rodericensis }\end{array}$ & $\begin{array}{c}\text { PAUlian, 1950: } \\
84 \text { (Aiolopus sp.) } \\
\text { PAULIAN, 1951: } \\
186 \text { (Aiolopus } \\
\text { sansibarus) } \\
\text { VIETTE, } 1967: 195 \\
*\end{array}$ & $\begin{array}{c}\text { Paulian, } 1989: 205 \\
\text { Aiolopus sansibara } \\
\text { (voir texte) } \\
*\end{array}$ & & $\mathrm{X}$ \\
\hline Oedaleus virgula & & $\mathrm{X}$ & & $\mathrm{X}$ \\
\hline Conipoda calcarata & & $\mathrm{X}$ & & $\begin{array}{c}\text { DirSh \& } \\
\text { DesCAMPS, } 1968: \\
267\end{array}$ \\
\hline Total & 4 & 7 & 1 & 6 \\
\hline
\end{tabular}

Peuplement des îles. - Sur les dix espèces d'Acrididae des îles Eparses :

- toutes sont présentes à Madagascar, excepté Diabolocatantops axillaris, qui est largement présent en Afrique continentale et dont la sous-espèce D. axillaris saucius est présente dans les régions côtières de Somalie et du Kenya, ainsi qu'aux Comores et à Aldabra (DESCAMPS \& WinTREBERT, 1969 ; Bolívar, 1912 ; Dirsh, 1956) ;

- près de la moitié (4 espèces) est propre à Madagascar (tableau II) ;

- la moitié (5 espèces) est présente à Madagascar et ailleurs (notamment en Afrique continentale et/ou aux Comores). Parmi ces espèces à large répartition, deux sont à la fois présentes sur l'archipel des Glorieuses, à Europa et à Juan de Nova : Cyrtacanthacris tatarica et Aiolopus thalassinus rodericensis.

En conséquence, la faune des Acrididae des îles éparses se compose d'espèces présentes sur d'autres îles de la région malgache. Aucun cas d'endémisme n'a été observé. 
Madagascar semble jouer un rôle prédominant dans le peuplement des îles Eparses. Cependant la présence aux Glorieuses de D. axillaris saucius suggère l'existence d'échanges entre cet archipel et les Comores.

Tableau II. - Distribution des Acrididae dans la région afro-malgache.

\begin{tabular}{|l|l|c|c|c|}
\hline \multicolumn{1}{|c|}{ Distribution } & \multicolumn{1}{|c|}{ Espèces } & $\begin{array}{c}\text { Afrique } \\
\text { continentale }\end{array}$ & Madagascar & Comores \\
\hline \multirow{2}{*}{ Large répartition } & Cyrtacanthacris tatarica & $\mathrm{X}$ & $\mathrm{X}$ & $\mathrm{X}$ \\
\cline { 2 - 5 } & Acrotylus patruelis & $\mathrm{X}$ & $\mathrm{X}$ & $\mathrm{X}$ \\
\hline \multirow{4}{*}{$\begin{array}{l}\text { Afrique, Madagascar } \\
\text { (endémiques) }\end{array}$} & Oedaleus virgula & $\mathrm{X}$ & $\mathrm{X}$ & \\
\hline & Catantopsis malagassus & & Endémique & \\
\cline { 2 - 5 } & Rhadinacris schistocercoides & & Endémique & \\
\cline { 2 - 5 } & Acrida madecassa & & Endémique & \\
\cline { 2 - 5 } $\begin{array}{l}\text { Madagascar, } \\
\text { Comores }\end{array}$ & Conipoda calcarata & & Endémique & \\
\hline Afrique, Comores & Catantopsis sacalava & & $\mathrm{X}$ & $\mathrm{X}$ \\
\cline { 2 - 6 } & Aiolopus thalassinus rodericensis & & $\mathrm{X}$ & $\mathrm{X}$ \\
\hline
\end{tabular}

Ecologie. - Mis à part Cyrtacanthacris tatarica qui fréquente des milieux arbustifs, les autres espèces se répartissent entre les habitats herbeux (prairie, bord de piste, savane, etc.) et les habitats sableux (dune, chemin sableux, etc.). La moitié des espèces vivent dans des habitats herbeux sans que l'on puisse mettre en évidence de spécificité à l'exception d'Acrida madecassa qui est plus particulièrement trouvé dans les hautes herbes de l'arrière-littoral (tableau III).

Parmi les quatre espèces associées aux habitats sableux, les deux espèces des Glorieuses (Conipoda calcarata, Oedaleus virgula) vivent dans les dunes sur le littoral tandis que les deux autres affectionnent les zones sableuses plus à l'intérieur des terres.

Tableau III. - Habitats fréquentés par les différentes espèces dans chacune des îles.

\begin{tabular}{|l|c|c|c|}
\hline Habitat & Europa & Glorieuses & Juan de Nova \\
\hline $\begin{array}{l}\text { Sable, } \\
\text { littoral }\end{array}$ & Acrotylus patruelis & $\begin{array}{c}\text { Conipoda calcarata } \\
\text { Oedaleus virgula }\end{array}$ & Catantopsis sacalava \\
\hline $\begin{array}{l}\text { Herbes, } \\
\text { savane }\end{array}$ & $\begin{array}{c}\text { Cyrtacanthacris tatarica } \\
\text { Rhadinacris schistocercoides }\end{array}$ & $\begin{array}{c}\text { Diabolocatantops axillaris } \\
\text { saucius } \\
\text { Aiolopus thalassinus rodericensis }\end{array}$ & $\begin{array}{c}\text { Acrida madecassa } \\
\text { Aiolopus thalassinus } \\
\text { rodericensis }\end{array}$ \\
\hline $\begin{array}{l}\text { Arbustes, } \\
\text { bush }\end{array}$ & Cyrtacanthacris tatarica (?) & Cyrtacanthacris tatarica & Cyrtacanthacris tatarica \\
\hline
\end{tabular}

\section{CONCLUSION}

Cette étude constitue la première synthèse concernant les Acrididae des îles Eparses. Les connaissances de ce groupe en sont enrichies : quatre espèces inédites sont signalées, les inventaires de chacune des îles sont établis, y compris celui de l'île du Lys, des données sur l'écologie sont mentionnées, une collection de référence est constituée et déposée au Muséum d'Histoire naturelle de la Réunion.

La faune se compose d'espèces de la zone malgache originaires pour la plupart de la Grande Ile. Cependant, l'étude des Acridiens montre également l'influence des Comores dans le peuplement des Glorieuses. 
Remerciements. - Nous remercions le Muséum d'Histoire Naturelle de la Réunion pour l'organisation des missions de RP, le laboratoire ECOMAR (Université de la Réunion) et l'IMEP (CNRS) pour l'organisation des missions de GC, Jean David (CNRS) pour les collectes complémentaires et nos collègues du Conservatoire botanique national de Mascarin pour l'identification des végétaux durant la durant la mission de 2011, Simon Poulain (MNHN) pour avoir recherché les spécimens des îles Eparses dans les collections du MNHN et Peter J. Schwendinger (MHNG) pour l'envoi de photographies des types de Conipoda. La mission d'AF, GC, JR et SG a bénéficié du financement de la fondation Linné du CNRS et de la rotation d'avril 2011 du navire de recherches Marion Dufresne dans les îles Eparses.

\section{AUTEURS CITÉS}

BoLÍvar I., 1912. - Orthoptera: Acrydiidae, Phasgonuridae, Gryllidae (Percy Sladen Trust Expedition to the Seychelles). Transactions of the Linnean Society of London, Zoology, (2) 15 : 263-292.

Descamps M. \& Wintrebert D., 1969. - Aperçu de l'Acridofaune comorienne. Annales de la Société entomologique de France (N. S.), 5 (3) : 537-568.

DiRsh V. M., 1956. - Preliminary revision of the genus Catantopsis Schaum and review of the group Catantopini (Orthoptera, Acrididae). Publicaçoes Culturais da Companhia de Diamantes de Angola, $28: 11-151$.

DirSh V. M. \& DesCAmPs M., 1968. - Insectes Orthoptères Acridoidea. Faune de Madagascar, 26 : 312 p.

Матуот P., 1998. - The orthopteroids of the Seychelles: a threatened island fauna. Journal of Insect Conservation, 2 (3-4) : 235-246.

Paulian R., 1950. - L'île Europa, une dépendance de Madagascar. Le Naturaliste malgache, 2 (2) : 77-85.

— 1951. - Les insectes de l'île Europa. Le Naturaliste malgache, 3 : 185-186. 1955. - Observations sur la faune terrestre de l'île Tromelin. Le Naturaliste malgache, 7 (1) : 1-7. 1989. - Les insectes des îles Glorieuses. L'entomologiste, 45 (4-5) : 203-208.

Saussure H. DE, 1884. - Prodromus Oedipodiorum - Insectorum ex ordine Orthopterorum. Mémoires de la société de Physique et d'Histoire naturelle de Genève, 28 (9) : 1-256.

— 1899. - Wissenschaftliche Ergebnisse der Reise in Madagaskar und Ostafrika in den Jahren 1889-95 von Dr A. Voeltzkow, Orthoptera. Abhandlungen der Senckenbergischen Naturforschenden Gesellschaft, 21 : $569-664$.

Viette P., 1967. - L'entomofaune de l'île Europa. Mémoires du Muséum national d'Histoire naturelle, N. S., (A) 41 [1966] : 191-209. 